\title{
Doença óssea adinâmica
}

\author{
Adynamic bone disease
}

\section{Autores:}

Rodrigo Bueno de Oliveira

Rosa Maria Affonso Moysés

Lillian Andrade da

Rocha

Aluizio Barbosa de Carvalho
1 Diagnóstico da doença óssea adinâmica (DOA)

1.1 Deve-se suspeitar de DOA em pacientes idosos, diabéticos, paratireoidectomizados, tratados por longos períodos com doses elevadas de sais de Ca e/ou calcitriol ou análogos da vitamina $\mathrm{D}$, que utilizam, por longos períodos, dialisato com concentrações elevadas de $\mathrm{Ca}(3,5$ $\mathrm{mEq} / \mathrm{L}$ ) e em uso de corticosteroides (Opinião).

1.2 O diagnóstico de DOA só pode ser confirmado por meio de biópsia óssea não descalcificada com análise histomorfométrica (Evidência).

1.3 Níveis séricos de paratormôniointacto (PTH) inferiores a $120 \mathrm{pg} / \mathrm{mL}$ sugerem o diagnóstico de DOA (Opinião).

1.4 Níveis elevados de fosfatase alcalina total (FA), em pacientes sem doenças hepáticas, ou de sua fração óssea, praticamente excluem DOA (Evidência).

1.5 Diante da suspeita de DOA, a intoxicação por alumínio deve ser excluída por meio do teste à desferroxamina ou da biópsia óssea com coloração para alumínio (Evidência).

\section{Tratamento da doença óssea adinâmica}

2.1 Pacientes com DOA devem restringir a ingestão de $\mathrm{Ca}$ por meio da dieta ou do uso de quelantes de $\mathrm{P}$ contendo Ca. Uma alternativa é o uso de quelantes de $\mathrm{P}$ isentos de $\mathrm{Ca}$, como o carbonato ou cloridrato de sevelamer, ou o carbonato de lantânio (Evidência).
2.2 O tratamento da DOA deve incluir a terapia com desferroxamina (quando houver intoxicação por alumínio), restrição do aporte de $\mathrm{Ca}$ (tanto por via oral quanto através do dialisato) e correção de fatores que colaboram para o aumento da resistência óssea ao PTH, como inflamação, desnutrição, hipotireoidismo e diabete melito (Evidência).

2.3 Nos pacientes com DOA, a concentração de $\mathrm{Ca}$ no dialisato deve ser de 2,5 ou 3,0 mEq/L. Dialisato com concentração de $\mathrm{Ca}$ de $3,5 \mathrm{mEq} / \mathrm{L}$ deve ser evitado (Opinião).

2.4 Nos pacientes com DOA e hipovitaminose $\mathrm{D}$, a reposição se faz com vitamina $\mathrm{D}_{2}$ ou $\mathrm{D}_{3}$ (Opinião).

2.5 No pós-operatório de pacientes submetidos à paratireoidectomia total com autoimplante, os níveis séricos de Ca iônico (Cai) devem ser mantidos no limite inferior dos valores de referência, com o objetivo de estimular a produção de PTH pelo enxerto (Opinião).

\section{RAcional}

Doença óssea adinâmica (DOA), também conhecida como doença óssea aplástica, se caracteriza por diminuição da formação óssea, assim como da matriz osteoide e do número de osteoblastos. A fibrose na medula óssea está reduzida ou ausente. Esse perfil histológico caracteriza a baixa remodelação óssea. ${ }^{1}$

A prevalência de DOA vem aumentando nas últimas décadas, apesar da redução das doenças de baixa remodelação ligadas à intoxicação alumínica. 
Nos pacientes incidentes em diálise, a prevalência pode atingir $23 \% .^{2}$ Em pacientes prevalentes em diálise peritoneal (DP) e em diabéticos, cerca de $67 \%$ apresentam DOA. ${ }^{1-3}$

Os fatores de risco classicamente associados a DOA são: idade avançada, diabete melito, hipotireoidismo, hipoparatireoidismo (geralmente secundário à paratireoidectomia), pacientes em uso de corticosteroides, tratados por longos períodos com doses elevadas de sais de $\mathrm{Ca}$ e/ou calcitriol ou análogos da vitamina $\mathrm{D}$ e intoxicação por alumínio. ${ }^{1}$ A DOA é geralmente pouco sintomática (com exceção dos pacientes com intoxicação por alumínio). No entanto, está associada a maior risco de calcificação vascular (CV) e fraturas ósseas. Tais complicações aumentam significativamente a morbidade e a mortalidade dos pacientes. ${ }^{4-8}$

O padrão-ouro para o diagnóstico de DOA é a biópsia óssea. ${ }^{1}$ Em caso de indisponibilidade da biópsia óssea, marcadores bioquímicos da remodelação óssea podem ser úteis. Assim, níveis séricos de PTH persistentemente reduzidos ou inferiores a 120 $\mathrm{pg} / \mathrm{mL}$ estão associados à DOA. Níveis de PTH persistentemente elevados, acima de $450 \mathrm{pg} / \mathrm{mL}$, geralmente afastam o diagnóstico de DOA. ${ }^{9}$ Níveis elevados de FA, em pacientes sem doenças hepáticas, ou de sua fração óssea, praticamente excluem DOA. ${ }^{10,11}$

Até o momento, não existem estudos em grande escala, prospectivos, randomizados e controlados sobre o tratamento da DOA. ${ }^{12} \mathrm{O}$ tratamento atual da DOA segue dois princípios básicos: redução da carga de Ca (via oral e dialisato) e aumento dos níveis séricos do PTH, o que permite a reversão da DOA em número significativo de pacientes. ${ }^{13,14}$

O nível sérico de Cai é o principal regulador da síntese e secreção de PTH. A redução da concentração de $\mathrm{Ca}$ no dialisato (tanto na diálise peritoneal quanto na hemodiálise) favorece o aumento dos níveis de PTH e, consequentemente, melhora da remodelação óssea. ${ }^{15-17}$ Dessa forma, pacientes com DOA devem receber quelantes de $\mathrm{P}$ isentos $\mathrm{de}^{\mathrm{Ca}}{ }^{1}$ e serem dialisatos com menor concentração de $\mathrm{Ca}$. $\mathrm{O}$ uso de teriparatide (PTH 1-34 recombinante humano) teoricamente deveria restaurar a remodelação óssea. Contudo, não existem estudos controlados com o uso dessa droga em pacientes com DRC. ${ }^{1}$

\section{REFERÊNCIAS}

1. Brandenburg VM, Floege J. Adinamic Bone Disease: Bone and Beyond. Nephrol Dial Tranplant. 2008; 3:135-47.

2. Sherrard DJ, Hercz G, Pei Y et al. The spectrum of bone disease in end-stage renal failure - an evolving disorder. Kidney Int. 1993; 43:436-42.
3. Spasovski GB, Bervoets AR, Behets GJ et al. Spectrum of renal bone disease in end-stage renal failure patients not yet on dialysis. Nephrol Dial Transplant. 2003; 18:1159-66.

4. Barreto DV, Barreto FC, Carvalho $\mathrm{AB}$ et al. Coronary calcification in hemodialysis patients: the contribution of traditional and uremia-related risk factors. Kidney Int. 2005; 67:1576-82.

5. London GM, Marty C, Marchais SJ et al. Arterial calcifications and bone histomorphometry in end-stage renal disease. J Am Soc Nephrol. 2004; 15:1943-51.

6. Ganesh SK, Stack AG, Levin NW et al. Association of elevated serum $\mathrm{PO}(4), \mathrm{Ca} \times \mathrm{PO}(4)$ product, and parathyroid hormone with cardiac mortality risk in chronic hemodialysis patients. J Am Soc Nephrol. 2001; 12:2131-8.

7. Avram MM, Mittman N, Myint MM et al. Importance of low serum intact parathyroid hormone as a predictor of mortality in hemodialysis and peritoneal dialysis patients: 14 years of prospective observation. Am J Kidney Dis. 2001; 38:1351-7.

8. Guh JY, Chen HC, Chuang HY et al. Risk factors and risk for mortality of mild hypoparathyroidism in hemodialysis patients. Am J Kidney Dis. 2002; 39:1245-54.

9. Torres A, Lorenzo V, Hernandez D et al. Bone disease in predialysis, hemodialysis, and CAPD patients: evidence of a better bone response to PTH. Kidney Int. 1995; 47:1434-42.

10. Schwarz C, Sulzbacher I, Oberbauer R. Diagnosis of renal osteodystrophy. Eur J Clin Invest. 2006; 36(Suppl 2):13-22.

11. Urena P, De Vernejoul MC. Circulating biochemical markers of bone remodeling in uremic patients. Kidney Int. 1999; 55:2141-56.

12. Martin KJ, Gonzalez EA. Metabolic bone disease in chronic kidney disease. J Am Soc Nephrol. 2007; 18:875-85.

13. Haris A, Sherrard DJ, Hercz G. Reversal of adynamic bone disease by lowering of dialysate calcium. Kidney Int. 2006; 70:931-7.

14. D'Haese PC, Spasovski GB, Sikole A et al. A multicenter study on the effects of lanthanum carbonate (Fosrenol) and calcium carbonate on renal bone disease in dialysis patients. Kidney Int. 2003; 63(Suppl):S73-S8.

15. Spasovski G, Gelev S, Masin-Spasovska J et al. Improvement of bone and mineral parameters related to adynamic bone disease by diminishing dialysate calcium. Bone 2007; 41:698-703.

16. Lezaic V, Pejanovic S, Kostic S et al. Effects of lowering dialysate calcium concentration on mineral metabolism and parathyroid hormone secretion: a multicentric study. Ther Apher Dial. 2007; 11:121-30.

17. Fujimori A, Yorifuji M, Sakai M et al. Low-calcium dialysate improves mineral metabolism in hemodialysis patients. Clin Nephrol. 2007; 67:20-4. 